\title{
A Study on the Importance of Anti Cyclic Cytrullinated Peptide Antibodies (Anti-CCP) and Rheumatoid Factor (RF) Assay in Detection of Patients with Rheumatoid Arthritis in North Eastern Part of India
}

\author{
Anindya Das ${ }^{1}$, Chimanjita Phukan ${ }^{1}$, Chitralekha Baruah ${ }^{2}$ \\ ${ }^{1}$ Department of Microbiology, Gauhati Medical College and Hospital, Guwahati, India \\ ${ }^{2}$ Department of Medicine, Gauhati Medical College and Hospital, Guwahati, India
}

Email address:

dranindyadas20@gmail.com (A. Das),chimanjitaphukan@gmail.com (C. Phukan), baruah.chitralekha@gmail.com (C. Baruah)

\section{To cite this article:}

Anindya Das, Chimanjita Phukan, Chitralekha Baruah. A Study on the Importance of Anti Cyclic Cytrullinated Peptide Antibodies (Anti$\mathrm{CCP}$ ) and Rheumatoid Factor (RF) Assay in Detection of Patients with Rheumatoid Arthritis in North Eastern Part of India. International Journal of Immunology. Vol. 4, No. 5, 2016, pp. 27-34.doi: 10.11648/j.iji.20160405.11

Received: October 15, 2016; Accepted: October 31, 2016; Published: November 23, 2016

\begin{abstract}
Objective: Rheumatoid arthritis (RA) is a polyarticular and systemic chronic inflammatory autoimmune disease, occurring throughout the world. The aim of the present study is to observe the role of anti-CCP antibody and Rheumatoid Factor (RF) assay in diagnosing patients of rheumatoid arthritis in north-eastern part of India, in the state of Assam and also to evaluate the individual and combined sensitivity and specificity of anti-CCP antibody and RF assay in diagnosing RA. Methods: A total of 88 cases presenting with history of polyarthritis were included in the study of which the study group comprised of 57 clinically diagnosed cases of rheumatoid arthritis and control group comprised of 31 patients of non-RA rheumatic diseases having joint pain. Individual sensitivity and specificity of the above tests, and also the combined specificity of the two tests and also the three tests were calculated and the results of the tests were compared with each other to examine the correlation between them. Results: Sensitivity of anti-CCP ELISA, RF IgM ELISA and RF Latex test was 91.23\%, 75.44\% and $40.35 \%$ with specificity of $70.97 \%, 67.74 \%$ and $90.32 \%$ respectively. If the three tests are combined then the combined specificity of the three tests is $99.59 \%$. One important observation was that out of 9 anti-CCP positive cases in the control group $(\mathrm{n}=31) 5$ cases were weakly positive (titer $6.25-20 \mathrm{U} / \mathrm{ml}$ ), considering cut-off value $6.25 \mathrm{U} / \mathrm{ml}$ as mentioned by the kit.If these weakly positive cases in the control group are considered as negative, then specificity of anti-CCP ELISA increases from $70.97 \%$ to $87.1 \%$ which is more acceptable. Conclusion: From this study it is observed that anti-CCP antibodies are highly sensitive and specific for diagnosing RA. If this test is combined with RF IgM ELISA and Latex test then combined specificity can be increased more than $99 \%$. However, weakly positive titers of anti-CCP antibodies are more prevalent in other non-RA rheumatic diseases in North-Eastern part of India.
\end{abstract}

Keywords: Anti-CCP Antibody, Rheumatoid Factor, Latex Agglutination Test, Combined Specificity, Resultant Sensitivity, Enzyme Linked Immunosorbent Assay (ELISA), Cut-Off Value

\section{Introduction}

Rheumatoid arthritis (RA) is a common autoimmune disease with a significant level of morbidity. [1] The morbidity and mortality it causes are the consequence of local and systemic inflammatory process that damage cartilage, bone and soft tissue, as well as blood vessels and viscera. [2]
Because early initiation of disease modifying treatments can significantly improve long term outcomes for patients with RA, there is a considerable motivation to accurately diagnose RA in patients with inflammatory arthritis, early in the course of the disease. [3] But if RA is not accurately diagnosed early in the course of the disease, then overenthusiastic initiation of treatment with disease modifying agents can cause a lot of harm to the patient because, these drugs have many side 
effects. The ability to identify those patients who will have progressive, erosive disease also remains an objective, because this subset of patients may benefit most from early aggressive treatment. [4]

The first autoantibody in RA, rheumatoid factor (RF), was described by Norwegian Dr Erik Waaler in 1937 and redescribed by Dr H.M. Rose and colleagues in 1948. It was not until the early 1960s that investigators in the field agreed that RFs are antibodies to Fc fragment of IgG. [5] They are probably the most studied antibody since their discovery by Waaler in 1937. [6] Although RFs can be detected in the sera of a majority of RA patients, RF has been observed in many other autoimmune diseases, such as, in systemic lupus erythematosus, mixed connective tissue disease and primary Sjogren syndrome, as well as in infectious diseases such as Mycobacterium tuberculosis, Lyme disease, and also in healthy people. So, not all rheumatoid factors cause disease, and many studies are recently going on to characterize the difference between "pathological" and "physiological" RFs. [7]

The need for a better laboratory marker with a higher disease-related specificity and sensitivity for RA was always evident and an ongoing research was going on over the past 30 years. A specific antibody for RA was first described by Nienhuis and Mandema (1964), which is called antiperinuclear factor (APF) as these antibodies combine with constituents of the keratohyaline granules which are located close to the nucleus of buccal mucosa cells of adult people. Hoet et al. in 1991 showed that APF had an acceptable sensitivity and much higher specificity in comparison to RF. However, the test was never used for routine testing due to practical inconvenience. The test actually required carefully selected buccal mucosal cells which were differentiated enough to contain the perinuclear factor. At the same time, experienced laboratory technicians were also required to perform the test based on indirect immunofluorescence and to recognize the different immunofluorescent patterns. [8]

In 1979, Young et al. reported that sera of RA patients contained another group of antibodies, named antikeratin antibodies (AKA) which bind to keratin-like structures in the cornified layer of stratum corneum. Hoet and van Venrooij in 1992 mentioned that specificity of AKA was comparable to APF. Kurki et al. in 1992 demonstrated that AKA also precede the onset of RA. [8] Sebbag et al. in 1995 showed that APF and AKA target the same antigen identified as the epithelial protein filaggrin (filament aggregating protein), which is actually involved in the organization of cytoskeletal structures. [9] During differentiation of epithelial cells several filaggrin subunits result from proteolytic cleavage of profilaggrin. In profilaggrin, about $20 \%$ of the basic arginine residues are converted into neutral citrulline residues by the enzyme peptidyl-arginine deiminase (PADI). [8] Four isoforms of PADI have been identified, known as PADI1, PADI2, PADI3 and PADI4. Schellekens et al. in 1998 documented that, citrullination is essential for the autoantigenicity of filaggrin. Citrullinated fillaggrin is the antigen targeted by APF and AKA. Based on that finding,
Schellenkens et al. developed an enzyme linked immunosorbent assay (ELISA) derived from the sequence of human filaggrin as substrate.[10]The assay was later improved as second generation anti-CCP test with a sensitivity of $70-80 \%$ and specificity of $95-98 \%$ in established rheumatoid arthritis and controls. [21]

The usefulness of anti-CCP antibodies to identify rheumatoid arthritis from other rheumatic diseases, presenting with joint pain has not yet been studied in patients of North-Eastern part of India. As we know that the incidence and prevalence of RA varies based on geographic location, both globally and among certain ethnic groups in a country, and as genetic factors play some role in causation of the disease, [29] the aim of the present study is to see the role of anti-CCP antibody and rheumatoid factor assay in diagnosing patients of rheumatoid arthritis in North-Eastern part of India, and also to evaluate the combined sensitivity and specificity of both anti-CCP antibody and rheumatoid factor assay in diagnosing RA.

\section{Materials and Methods}

\subsection{Study Design}

The present study was a hospital based observational/case control study, carried out over a period of one year from August 2014 to July 2015, that was conducted on a total of 88 patients presenting with history of polyarthritis in the Rheumatology OPD of the Gauhati Medical College and Hospital (GMCH), which caters to a large outpatient population in the North Eastern part of India. The study was conducted in two groups of which the study group was composed of 57 patients who attended the Rheumatology OPD for treatment and were diagnosed as having rheumatoid arthritis by the attending physician. The diagnosis was based on clinical features and "The New 2010American College of Rheumatology (ACR) and the European League Against Rheumatism (EULAR) criteria" for the diagnosis of rheumatoid arthritis and finally on the expert opinion of attending physician of Rheumatology OPD. The control group was composed of 31 patients attending the Rheumatology OPD of GMCH, and was diagnosed as having non-RA rheumatic diseases with joint pain like systemic lupus erythematosus (SLE), mixed connective tissue disease (MCTD), ankylosing spondylitis (AS) and spondyloarthropathy, gouty arthritis, osteoarthritis (OA), reactive arthritis and other non-specific polyarthritis. The study group consisted of $42(73.68 \%)$ females and 15 $(26.32 \%)$ males, while the control group consisted of 12 (38.71\%) females and $19(61.29 \%)$ males. The female to male ratio in the study group was 2.8: 1 , whereas in the control group males were more than the females, with a male to female ratio of 1.6: 1 .

\subsection{Ethical Considerations}

Ethical clearance was obtained from Institutional Ethics Committee (IEC), Gauhati Medical College and Hospital, 
Guwahati, India (reg. no. MC/02/2015/100). Participation in the study was voluntary and a signed consent form was obtained from all the patients. The study results were returned to the patients and incorporated into their medical records.

\subsection{Sample Collection}

About $5 \mathrm{ml}$ of venous blood was collected aseptically in a sterile vial from each patient of both study and control groups. The vial was left at room temperature and the blood was allowed to clot for separation of serum. Then serum was separated by centrifuging the sample vials in a centrifuge machine at 3000 revolutions per minute (rpm) for 5 minutes in Microbiology laboratory. The separated serums were then transferred to sterile vials and, labeled properly with serial numbers to identify them properly later on. The serum samples were stored at $-80^{\circ} \mathrm{C}$ till the assay was done.

\subsection{Laboratory Methods}

Anti-CCP antibodies were detected by ELISA, using a commercial kit (Omega Genesis EDRA bioMerieux, Cambridgeshire,UK) and according to the manufacturer's instructions, expressed in optical density (OD) along y-axis and corresponding antibody titer of the standards, plotted along $\mathrm{X}$-axis of the curve, as shown in Fig.1. Rheumatoid Factor (RF) assay was done by Latex Agglutination Slide Test with RHELAX-RF reagent Kit,( Tulip Diagnostics (P) Ltd. India) and subsequently the sera were examined for IgM Rheumatoid Factor by Autostat ${ }^{\mathrm{TM}}$ II Rheumatoid Factor IgM ELISA Kit (Hycor Biomedical, Garden Grove, California, USA). The tests were done according to manufacturer's instructions.

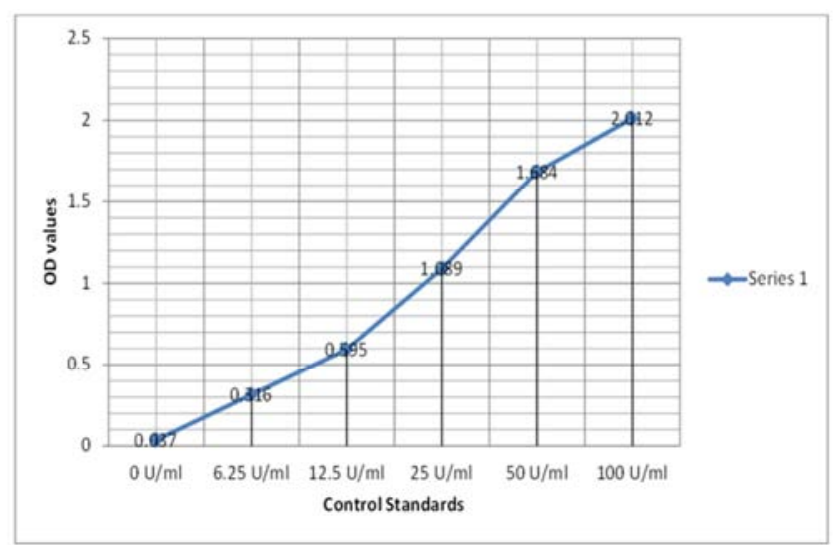

Fig. 1. Titer of Control Standards and their OD value producing the curve

\subsection{Statistical Analysis}

Then statistical analyses of the test results were done by using the software GraphPadinStat and online Med Calc. statistical calculator. The distribution of laboratory test results were compared between study group and control group using the chi- square test or the Fisher's exact test,where necessary. Two-sided $\mathrm{p}<0.05$ was considered significant. Diagnostic value of the tests were described by determining the sensitivity, specificity, positive predictive value (PPV) and negative predictive value (NPV) obtained with the cut-off value mentioned by the respective commercial kits. Assuming the prevalence of RA in the community as $0.5 \%$, corrected PPV and NPV was calculated using Bayes' theorem. Correlation between variables was assessed by Spearman's correlation coefficient and Pearson correlation.

\section{Results}

Anti-CCP antibody was positive in 52(91.23\%) out of 57 cases in the study group, and was negative in only $5(8.77 \%)$ out of 57cases in the study group. In case of control group the picture was different as anti-CCP antibody was positive in $9(29.03 \%)$ out of 31 cases in this group. One important observation was that, out of 9 anti-CCP positive cases in the control group, 5 cases were weakly positive (titer $<3 \times$ cutoff value) for anti-CCP antibody. Anti-CCP antibody was negative in 22 out of $31(70.97 \%$ ) cases in the control group. When the serological profile of anti-CCP antibody in the study group and control group was compared it was found to be significant. $(\mathrm{p}<0.0001)$ The sensitivity, specificity, positive predictive value and negative predictive value of the test was $91.23 \%, 70.97 \%, 75.77 \%$ and $89 \%$ respectively. Here, specificity of anti-CCP antibody test is showing lower value, though the sensitivity of the test is high.

One important thing that is observed in this study, that if 5 weakly positive cases (titer $<3 \times$ cut-off value) in the control group are excluded from the 9 positive cases in the control group and considered as negative, then False Positive cases become 4. In that case, 52 patients are True Positive for antiCCP antibody, 5 patients are False Negative for anti-CCP antibody, 4 patients are False Positive for anti-CCP antibody and $22+5=27$ patients are True Negative for anti-CCP antibody, which has been shown in Table1. When the serological profile of anti-CCP antibody is modified by considering weakly positive cases as negative and the study group and control group is compared, it is found to be significant. $(\mathrm{p}<0.0001)$.

Table 1. Modification of serological results, considering weakly positive cases as negative.

\begin{tabular}{|c|c|c|}
\hline Test & Study group & Control group \\
\hline anti-CCP positive & 52(true positive) & $\begin{array}{l}\text { (positive }- \text { weakly positive } \\
=\text { false positive })(9-5=4)\end{array}$ \\
\hline anti-CCP negative & 5 (false negative) & $22+5=27$ (true negative) \\
\hline Total $(\mathrm{n}=88)$ & 57 & 31 \\
\hline \multicolumn{3}{|c|}{$\chi^{2}=49.902$, degree of freedom $=1, p<0.0001$} \\
\hline
\end{tabular}

Here, due to this modification, the sensitivity and specificity of test are showing more accurate results, which are $91.23 \%$ and $87.10 \%$ respectively. Though according to the kit, cut-off value is $6.25 \mathrm{U} / \mathrm{ml}$, in the control group significant numbers of patients are becoming weakly positive (titer between $6.25-20 \mathrm{U} / \mathrm{ml}$ ) for anti-CCP antibody test. So if the 5 weakly positive $(6.25-20 \mathrm{U} / \mathrm{ml})$ cases in the control group are considered as negative, then specificity of the test is increasing significantly from $70.97 \%$ to $87.10 \%$. 
Modification of the results can be done in another way by increasing the cut-off value from $6.25 \mathrm{U} / \mathrm{ml}$ to $20 \mathrm{U} / \mathrm{ml}$, in order to exclude the weakly positive cases from the positive cases of the control group who are having non-RA other rheumatic disorders. So after increasing cut-off value to 20U/ml, 51 patients are True Positive for anti-CCP antibody,6 patients are False Negative for anti-CCP antibody, 4 patients are False Positive for anti-CCP antibody and 27 patients are True Negative for anti-CCP antibody which is shown in Table 2.

Table 2. Modification of serological results by increasing cutoff value from $6.25 \mathrm{U} / \mathrm{ml}$ to $20 \mathrm{U} / \mathrm{ml}$.

\begin{tabular}{lll}
\hline Test & Study group & Control group \\
\hline anti-CCP positive & 51(true positive) & 4 (false positive) \\
anti-CCP negative & 6 (false negative) & 27(true negative) \\
Total $(\mathrm{n}=88)$ & 57 & 31 \\
$\chi^{2}=47.016$, degree of freedom $=1, \mathrm{p}<0.0001$ & \\
\hline
\end{tabular}

Then sensitivity, specificity, positive predictive value and negative predictive value of the test is $89.47 \%, 87.1 \%$, $87.39 \%$ and $89.21 \%$ respectively. Therefore, if the cut-off value is increased to $20 \mathrm{U} / \mathrm{ml}$ ( $3 \times$ previous cut-off), then specificity of the test is increasing significantly from $70.97 \%$ to $87.1 \%$ although, the sensitivity is reduced slightly from $90.23 \%$ to $89.47 \%$.

In the control group, distribution of anti-CCP positive (titer $>20 \mathrm{U} / \mathrm{ml}$ ), anti-CCP weakly positive (titer $6.25-20$ $\mathrm{U} / \mathrm{ml}$ ) and anti-CCP negative (titer $<6.25 \mathrm{U} / \mathrm{ml}$ ) cases in nonRA other rheumatic disease patients, like Ankylosing spondylitis, SLE, Spondyloarthropathy, mixed connective tissue disease(MCTD), Osteoarthritis(OA), Reactive arthritis, Gout + Osteoarthritis and Non specific arthritis are shown in Table 3.

Table 3. Number of Anti-CCP (+), Anti-CCP weakly (+), Anti-CCP (-) cases in different non-RA rheumatic diseases.

\begin{tabular}{|c|c|c|c|c|}
\hline Disease & Total & anti-ccp $(+)$ & anti-ccp weakly(+) & anti-ccp (-) \\
\hline Ankylosing spondylitis & 8 & 1 & 1 & 6 \\
\hline Systemic lupus erythematosus (SLE) & 6 & 2 & 0 & 4 \\
\hline Spondyloarthropathy & 2 & 0 & 1 & 1 \\
\hline mixed connective tissue disease (MCTD) & 2 & 0 & 0 & 2 \\
\hline Osteoarthritis (OA) & 2 & 0 & 0 & 2 \\
\hline Reactive arthritis & 2 & 0 & 0 & 2 \\
\hline Gout + Osteoarthritis & 2 & 0 & 1 & 1 \\
\hline Non specific arthritis & 7 & 1 & 2 & 4 \\
\hline Total & 31 & 4 & 5 & 22 \\
\hline
\end{tabular}

The sensitivity and specificity of Rheumatoid Factor Latex agglutination test is $40.35 \%$ and $90.32 \%$ respectively. Positive Predictive Value (PPV) and Negative Predictive Value (NPV) of the test is $80.65 \%$ and $60.22 \%$ respectively. Here sensitivity of RF Latex agglutination test is $40.35 \%$, but the sensitivity of anti-CCP antibody test is $91.23 \%$ and $89.47 \%$ (after increasing cut-off value). The difference between the sensitivity of the two tests is statistically extremely significant with $\mathrm{p}$ value of $<0.0001$ (using Fisher's exact test).

The sensitivity and specificity of Rheumatoid Factor IgM ELISA is $75.44 \%$ and $67.74 \%$ respectively and Positive Predictive Value (PPV) and Negative Predictive Value (NPV) of the test is $70.07 \%$ and $73.31 \%$ respectively. Here sensitivity of RF IgM ELISA (75.44\%) is higher than the sensitivity of RF Latex agglutination test $(40.35 \%)$ which is statistically extremely significant with $\mathrm{p}$ value of 0.0003 using Fisher's exact test. However specificity of RF IgM ELISA $(67.74 \%)$ is lower than the specificity of RF Latex agglutination test $(90.32 \%)$ which is statistically not quite significant $(\mathrm{p}=0.0586)$.

In the present study, the correlation coefficient for antiCCP ELISA and RF Latex test is 0.3551 (95\% CI, 0.1573 to $\left.0.5255, r^{2}=0.1261\right)$. The two tailed $p$ value is 0.0007 which is highly significant. The correlation coefficient for anti-CCP ELISA and RF IgM ELISA is 0.6318 (95\% CI, 0.4867 to $\left.0.7430, r^{2}=0.3992\right)$. The two tailed $\mathrm{p}$ value is $<0.0001$ which is extremely significant. The correlation coefficient for RF
IgM ELISA and RF Latex test is 0.7679 (95\% CI 0.6655 to $\left.0.8420, r^{2}=0.5897\right)$. The two tailed $\mathrm{p}$ value is $<0.0001$ which is extremely significant.

In the present study, anti-CCP antibody test is positive in 95.65\% RF Latex positive patients versus $88.23 \%$ RF Latex negative patients.(Fig. 2)

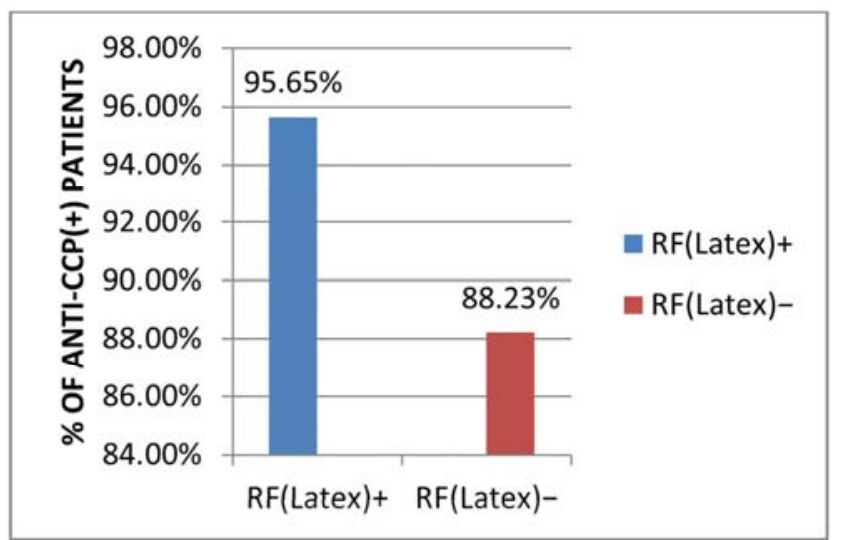

Fig. 2. Percentage of Anti-CCP(+) patients in RF Latex test positive and negative patient.

Similarly anti-CCP antibody is positive in $97.67 \%$ RF IgM ELISA positive patients versus $71.42 \%$ RF IgM ELISA negative patients. (Fig. 3) So we can say that anti-CCP antibody can be found more often in RF Latex test positive and RF IgM ELISA positive patients. 


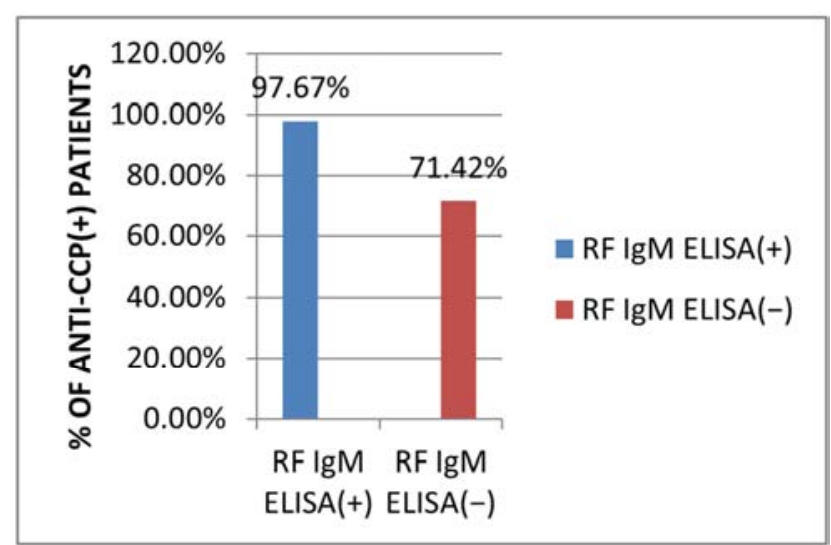

Fig. 3. Percentage of Anti-CCP(+) patients in RF IgM ELISA positive and negative patients.

Similarly Latex test is positive in $42.30 \%$ anti-CCP positive patients versus $20 \%$ of anti-CCP negative patients. (Fig. 4) So we can also say that RF Latex test can be found more often in anti-CCP positive patients.

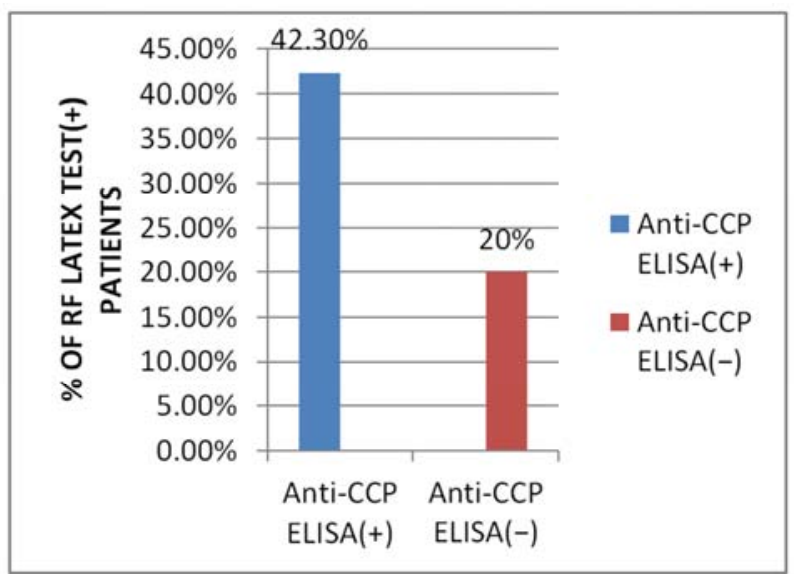

Fig. 4. Percentage of RF Latex test (+) patients in Anti-CCP positive and negative patients.

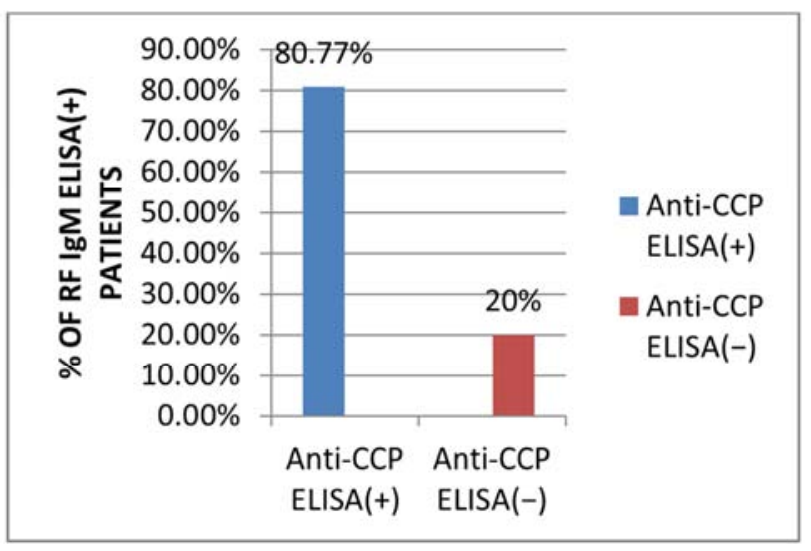

Fig. 5. Percentage of RF IgM ELISA(+) patients in Anti-CCP positive and negative patients.

RF IgM ELISA is positive in $80.77 \%$ anti-CCP positive patients versus $20 \%$ of anti-CCP negative patients. (Fig. 5) So we can also say that RF IgM ELISA can be found more often in anti-CCP positive patients.
In normal clinical practice, the results of two independent tests can be combined to be more confident of the diagnosis. The two tests can be combined to increase the specificity by using following formula:

Specificity of combined test $=1-(1-$ specificity of test 1$)$ $\times(1-$ specificity of test 2$)$

Thus by combining anti-CCP assay and Rheumatoid Factor Latex test, the combined specificity is increasing to $98.75 \%$ which indicates that, in a polyarthritis patient if both anti-CCP and RF(Latex test) is negative then there is $98.75 \%$ chance that the patient is negative for rheumatoid arthritis. But one drawback in combining these two tests in this way is that, here the resultant sensitivity of the two tests calculated by the following formula is $36.81 \%$, which is less than the individual sensitivity of anti-CCP and RF (Latex test) assay.

Resultant combined sensitivity $=$ sensitivity of test $1 \times$ sensitivity of test 2

Similarly if anti-CCP antibody test is combined with RF IgM ELISA then combined specificity of the two tests is $95.81 \%$ and the resultant sensitivity is $68.82 \%$. Individual and combined sensitivity and specificity of all the tests are shown below (Table 4).

Table 4. Individual \& combined sensitivity \& specificity of different tests in the present study.

\begin{tabular}{lll}
\hline Test & Sensitivity & Specificity \\
\hline Anti-CCP IgGELISA (after modification) & $91.23 \%$ & $87.10 \%$ \\
RF IgMELISA & $75.44 \%$ & $67.74 \%$ \\
RF Latex test & $40.35 \%$ & $90.32 \%$ \\
Combined anti-CCP ELISA + RF IgM ELISA & $68.82 \%$ & $95.81 \%$ \\
Combined anti-CCP ELISA + RF Latex test & $36.81 \%$ & $98.75 \%$ \\
\hline
\end{tabular}

After comparing all the results it is seen that combining these two results can more efficiently diagnose rheumatoid arthritis because, if a patient is negative for both anti-CCP and RF assay there is $95-98 \%$ chance of the patient not having rheumatoid arthritis though the resultant sensitivity is reduced.

Similarly by combining the anti-CCP ELISA, RF IgM ELISA and RF (Latex test), the combined specificity of the three tests can be calculated using the following formula:

Specificity of three combined tests $=1-(1-$ specificity of test 1$) \times(1-$ specificity of test 2$) \times(1-$ specificity of test 3$)$

Combined specificity of the three tests is $99.59 \%$, which means that if a patient is negative for all the three tests of anti-CCP ELISA, RF IgM ELISA and RF Latex test, then we may be $99.59 \%$ sure that the patient is not having rheumatoid arthritis, though the resultant sensitivity becomes $27.77 \%$.

\section{Discussion}

The present study was done to compare the diagnostic utility of anti-CCP antibody and RF assay in RA patients in comparison to those with non-RA other rheumatic diseases. Several studies have shown that anti-CCP antibody test is highly specific and sensitive for diagnosing RA, and could be detected early in the disease course.

In the present study, sensitivity and specificity of 
Rheumatoid Factor (Latex agglutination test) is $40.3 \%$ and $90.32 \%$ respectively. In another study conducted by Saraux et al. sensitivity of RF Latex test was $45 \%$. [11] In a different study conducted by Binesh et al. (2014), it was observed that the sensitivity of RF Latex test was $46.24 \%$ and the specificity was $90.29 \%$. [12] Aflaki et al. reported similar values of RF Latex test. [13] In the present study, sensitivity and specificity of Rheumatoid Factor IgM ELISA is $75.44 \%$ and $67.74 \%$ respectively. In a separate study conducted by Swedler et al. sensitivity and specificity of RF IgM ELISA was $91 \%$ and $76 \%$ respectively. [14] In another study conducted by Bas et al. sensitivity and specificity of Rheumatoid Factor IgM ELISA was $73 \%$ and $82 \%$ respectively. [15]

In the present study, sensitivity of RF IgM ELISA $(75.44 \%)$ is better than the sensitivity of RF Latex test $(40.3 \%)$ which is statistically extremely significant $(\mathrm{p}=$ 0.0003 ). Swedler et al. has also mentioned that sensitivity of RF IgM ELISA is better than the sensitivity of RF Latex test. [14] Niewold et al. has mentioned that, in several studies it has been observed that rheumatoid factor showed a variable sensitivity of $31 \%$ to $54 \%$ and specificity of $91 \%$ to $93 \%$ for the eventual diagnosis of RA when the test was done at first presentation, although rheumatoid factor was one of the criteria for the diagnosis of RA in these studies. Some of the variability in the sensitivity and specificity between studies may relate to slightly different cut-off points for positivity, and differences in disease duration, severity and other clinical characteristics of the groups being tested. [16]

In the present study, sensitivity and specificity of anti-CCP antibody is $91.23 \%$ and $70.97 \%$ respectively. In another study conducted by Gupta R et al. (2009) sensitivity and specificity of anti-CCP antibody was $85 \%$ and $90.19 \%$ respectively. [17] In a study conducted by Schellekens et al. (2000), sensitivity and specificity of anti-CCP antibody was $68 \%$ and $98 \%$ respectively. [18] Goldbach-M. et al. (2000) reported that sensitivity and specificity of anti-CCP antibody was 50\% and 90\% respectively. [19] According to Bizzaro et al. (2001) sensitivity and specificity of anti-CCP antibody was $41 \%$ and $98 \%$ respectively. [20] Walther J. et al. has mentioned that accumulated data from 164 studies published between 2002 and 2010 showed that sensitivity of anti-CCP antibody was $61-75 \%$ and specificity was 94-99\%. [21] Suzuki et al. (2003) reported that sensitivity and specificity of anti-CCP antibody was $88 \%$ and $89 \%$ respectively. [22] So it is quite obvious that the present study is showing reduced specificity of anti-CCP antibody test in comparison to the other studies, and it is mainly due to the large number of antiCCP positive cases $(29.03 \%)$ in the control group who are having non-RA other rheumatic diseases.

In the present study it has been observed that in the nonRA control group of patients, anti-CCP antibody was positive in 9 out of 31(29.03\%) cases. According to Walther J. et al. accumulated data from 164 studies published between 2002 and 2010 showed that $6.0 \%$ non-RA other rheumatic polyarthritis patients were positive for anti-CCP antibody. [21]
There may be several explanations for the difference between reported sensitivity and specificity of anti-CCP antibody test in different studies. One explanation for the discrepancy is that the differences in the patient populations (mainly disease duration) among these studies might have some influence on the results. Another study showed that the specificity and sensitivity of anti-CCP antibodies may depend on the patient's race. [23]

Another important observation in the present study was that, out of 9 anti-CCP positive cases in the control group 5 cases were weakly positive (titer $<3 \times$ cut-off value) for antiCCP antibody. Their anti-CCP titer is within the range of $6.25-20 \mathrm{U} / \mathrm{ml}$. If 5 weakly positive cases $(6.25-20 \mathrm{U} / \mathrm{ml})$ in the control group are excluded from the 9 positive cases in the control group and considered as negative, then the specificity of the test becomes $87.10 \%$ which is quite acceptable.

Explanation in favor of this modification is that people of North-Eastern part of India are genetically related to Mongolian, Chinese and Japanese people. In a large cohort study in Japan, it has been observed by Suzuki A, et al. that single nucleotide polymorphism of Peptidyl-arginine deiminase (PADI) gene is closely related to rheumatoid arthritis patients. [24] This as sociation has been confirmed in other Asian populations, but not in Western Europe. [25] So single nucleotide polymorphism of PADI gene may be common in North-Eastern part of India. Vossenaar, E. R. et $a l$. has mentioned that induction of PADI expression and citrullination of peptides are not specific to RA and can occur in many inflammatory settings. [26] So anti-CCP antibodies may be found in excess amount in other autoimmune inflammatory disorder. Although cross-reactivity of anti-CCP antibody with multiple citrullinated epitopes may occur, and could explain the recognition of multiple epitopes by a single antibody, the majority of anti-CCP antibodies seem to display distinct antigen-binding specificities. [21] Furthermore, as single nucleotide polymorphism may be common in this part of India, so weakly positive level of anti-CCP antibody may be found in other non-RA polyarthritis patients, and low positive level of anti-CCP antibody may vary from region to region in different ethnic groups of people. So in this part of India, if a patient has clinical findings suggestive of RA and anti-CCP titer at least three times higher than the cut-off value mentioned by the manufacturers, then we can be more confident about RA. But low positive titer of anti-CCP may be present in other type of arthritis patients.

In the present study anti-CCP antibody is positive in 97.67\% RF IgM ELISA positive patients versus $71.42 \% \mathrm{RF}$ IgM ELISA negative patients. So we can say that anti-CCP antibody can be found more often in RF IgM ELISA positive patients. Another study conducted by Saraux et al. also reported that anti-CCP antibody can be found more often in Rheumatoid Factor positive patients. [11] RF IgM ELISA is positive in $80.77 \%$ anti-CCP positive patients versus $20 \%$ of anti-CCP negative patients. So we can also say that RF can be found more often in anti-CCP positive patients. In a similar study Serdaroğlu et al. (2008) reported that RF is 
positive in $90 \%$ of anti-CCP positive (18 out of 20) patients, and in $40 \%$ of anti-CCP negative ( 8 out of 20 ) patients. [27]

In the present study, if anti-CCP antibody test is combined with RF IgM ELISA, then combined specificity is $95.81 \%$. In that case resultant sensitivity of the two tests is $68.82 \%$. If anti-CCP antibody test is combined with RF Latex test, then combined specificity is $98.75 \%$. In that case resultant sensitivity of the two tests is $36.81 \%$. Similarly by combining the anti-CCP ELISA, RF IgM ELISA and RF Latex test, the combined specificity of the three tests is $99.59 \%$. Parikh et al. has also shown that to make diagnosis of a disease more accurately, three diagnostic tests can be combined. [28]

\section{Conclusion}

This study showed that anti-CCP antibody assay can help significantly in diagnosis of RA. If anti-CCP antibody is combined with RF assay then RA can be more accurately diagnosed. However one problem of anti-CCP antibody test is determination of its cut-off value. The citrullination of arginine is under the control of PADI gene and in some studies genetic polymorphism of PADI gene has been found to be closely associated with rheumatoid arthritis in Asian people, particularly in Japanese people. The current study has been done in North Eastern part of India where people are genetically related to Mongolian, Chinese, Japanese people; so genetic polymorphism of PADI gene may be more prevalent in this region. In this study weakly positive titer (6.25-20U/ml) of anti-CCP antibody has been found in many other non-RA rheumatic diseases, and genetic polymorphism of PADI gene may be a cause behind this, which needs further studies. Correct determination of cut-off value is extremely important in predicting the accuracy of anti-CCP antibody assay. Cut-off value may vary from region to region and further study is needed to state this precisely. The combined anti-CCP and RF assay is more helpful than only anti-CCP assay in diagnosing RA accurately.

\section{Acknowledgements}

All the staffs of the Department of Microbiology and the Department of Medicine of Gauhati Medical College and Hospital,Guwahati, where this study was carried out.

\section{References}

[1] Arnett FC, et al. The American Rheumatism Association 1987 revised criteria for the classification of Rheumatoid Arthritis. Arthritis Rheum 1988; 31: 315-324.

[2] De Vries-Bouwastra JKD, Breedveld BAC, Ferdinand C. Biologics in early Rheumatoid Arthritis. Rheum Dis Clin North Am 2005; 31: 745-762

[3] O'Dell JR. Treating Rheumatoid Arthritis early: a window of opportunity? Arthritis Rheum 2002;46:283-285.

[4] Lee DM, Schur PH. Clinical utility of the anti-CCP assay in patients with rheumatic disease. Ann Rheum Dis 2003;

\section{2:870-874.}

[5] Milgrom F. Development of rheumatoid factor research through 50 years. Scand J Rheumatol Suppl. 1988; 75: 2-12.

[6] A J Soltys, J S Axford. Rheumatoid factors: where are we now?Ann Rheum Dis 1997; 56:285-286.

[7] Brian J. Sutton, Adam L. Corper, Maninder K. Sohi, Roy Jefferis, Dennis Beale, Michael J. Taussig. The Structure of a Human Rheumatoid Factor Bound to $\operatorname{IgG} \mathrm{Fc}$. Glycoimmunology 2Advances in Experimental Medicine and Biology 1998; 435: 41-50.

[8] M. Herold, V. Boeser, E. Russe, \& W. Klotz. Anti-CCP: History and its usefulness. Clinical \& Developmental Immunology 2005; 2: 131-135.

[9] Sebbag M, et al. The antiperinuclear factor and the so-called antikeratin antibodies are the same rheumatoid arthritis specific autoantibodies. J Clin Invest 1995; 95: 2672-2679.

[10] Schellekens GA, et al. Citrulline is an essential constituent of antigenic determinants recognized by rheumatoid arthritis specific autoantibodies. J Clin Invest 1998; 101: 273-281.

[11] Alain Saraux et al. Value of Antibodies to Citrullinecontaining Peptides for Diagnosing Early Rheumatoid Arthritis. The Journal of Rheumatology 2003; 30: 12.

[12] Fariba Binesh, Hossein Soleimani Salehabadi, Nasim Behniafard, Kian Ranginkaman and NasrinBehniafard. A Comparative Assessment of the Diagnostic Value of Anticyclic Citrullinated Peptide Antibodies and Rheumatoid Factor in Rheumatoid Arthritis. J Clin Exp Pathol 2014; 4: 158.

[13] Aflaky E, Shenavandeh S, Ashraf MJ. A comparison of performance of anti-cyclic citrullinated peptide 2 and citrullinated protein antibodies in the diagnosis of rheumatoid arthritis in Iranian patients. Rheumatol Int 2010; 30: 461-466.

[14] Swedler W, Wallman J, Froelich CJ, Teodorescu M. Routine measurement of $\operatorname{IgM}, \operatorname{IgG}$, and $\operatorname{IgA}$ rheumatoid factors: high sensitivity, specificity, and predictive value for rheumatoid arthritis. J Rheumatol. 1997; 6:1037-44.

[15] S. Bas, S. Genevay, O. Meyer and C. Gabay. Anti-cyclic citrullinated peptide antibodies, IgM and IgA rheumatoid factors in the diagnosis and prognosis of rheumatoid arthritis. Rheumatology 2003; 5: 677-680.

[16] T. B. Niewold, M. J. Harrison, and S. A. Paget. Anti-CCP antibody testing as a diagnostic and prognostic tool in rheumatoid arthritis. Q J Med 2007; 100:193-201.

[17] Gupta, Rajiv; Thabah, Molly M.; Aneja, Ritu; Kumar, Ashok; Varghese, Titus; Chandrasenan, P. J. Usefulness of Anti-CCP antibodies in rheumatic diseases in Indian patients. Indian Journal of Medical Sciences.2009; 3:92.

[18] Schellekens GA, Visser H, de Jong BA, van den Hoogen FH, HazesJM, Breedveld FC, van Venrooij WJ. The diagnostic properties of rheumatoid arthritis antibodies recognizing a cycliccitrullinated peptide. Arthritis Rheum 2000; 43:155163.

[19] Goldbach-Mansky R, Lee J, McCoy A, Hoxworth J, YarboroC,Smolen JS, Steiner G, Rosen A, Zhang C, Menard HA, Zhou ZJ, Palosuo T, Van Venrooij WJ, Wilder RL, KlippelJH, Schumacher HR Jr, El-Gabalawy HS. Rheumatoid arthritis associated autoantibodies in patients with synovitis of recent onset. Arthritis Res. 2000; 2:236-243. 
[20] Bizzaro N, Mazzanti G, Tonutti E, Villalta D, Tozzoli R. Diagnostic accuracy of the anti-citrulline antibody assay for rheumatoid arthritis. ClinChem 2001; 47:1089-1093.

[21] Walther J, van Venrooij, Joyce J, B. C. van Beers and Ger J. M. Pruijn. Anti-CCP antibodies: the past, the present and the future. Nat. Rev. Rheumatol2011; 7: 391-398.

[22] Suzuki K, Sawada T, Murakami A, Matsui T, Tohma S, NakazonoK, Takemura M, Takasaki Y, Mimori T, Yamamoto $\mathrm{K}$. High diagnostic performance of ELISA detection of antibodies to citrullinated antigens in rheumatoid arthritis. Scand J Rheumatol 2003; 32: 197-204.

[23] Mimori T. Clinical significance of anti-CCP antibodies in rheumatoid arthritis. Intern Med 2005; 44: 1122-1126.

[24] Suzuki A, et al: Functional haplotypes of PADI4, encoding citrullinating enzyme peptidyl arginine deiminase4, are associated with rheumatoid arthritis. Nat Genet.2003; 34: 395.
[25] Barton A, et al: A functional haplotype of the PADI4gene associated with rheumatoid arthritis in a Japanese population is not associated in a United Kingdom population. Arthritis Rheum. 2004; 50: 1117.

[26] Vossenaar, E. R. et al. The presence of citrullinated proteins is not specific for rheumatoid synovial tissue. Arthritis Rheum 2004; 50: 3485-3494.

[27] Münevver Serdaroğlu, Haşim Çakırbay, Orhan Değer, Sevil Cengiz, and Sibel Kul. The association of anti-CCP antibodies with disease activity in rheumatoid arthritis. Rheumatol Int. 2008;10: 965-970.

[28] Rajul Parikh, Annie Mathai, Shefali Parikh, G Chandra Sekhar, and Ravi Thomas, Understanding and using sensitivity, specificity and predictive values. Indian J Ophthalmol. 2008; 1: 45-50.

[29] Dennis L. Kasper, et al. Harrison's Principles of Internal Medicine, 19th edition, vol 2, 380:2136-2149. 